TP Periodica Polytechnica Electrical Engineering and Computer Science

60(4), pp. 211-216, 2016

DOI: 10.3311/PPee.9711

Creative Commons Attribution (i)

RESEARCH ARTICLE

\section{Influence of Topology on Performance of Organic Electrochemical Transistor}

\author{
Lukas Mracek ${ }^{1 *}$, Tomas Syrovy ${ }^{2}$, Silvan Pretl ${ }^{1}$, Stanislav Nespurek ${ }^{1}$, \\ Ales Hamacek ${ }^{1}$
}

Received 07 July 2016; accepted after revision 10 August 2016

\begin{abstract}
Organic electrochemical transistors (OECTS) with two topologies and three ionic liquid electrolytes were analyzed to get information about their influence on the selected transistor's characteristics. The channel of the OECT device was based on conductive polymer poly(3,4-ethylenedioxy-thiophene) poly(styrene sulfonate) (PEDOT:PSS). Dynamic characteristics were measured and analyzed. From the results follow that in the case of the double GATE configuration both the rise and decay current components contain diffusion contribution which limits the use of OECT in switching applications. Besides the influence of the OECT topology, the used electrolytes significantly affect transistors characteristics. Ionic liquid 1-butyl-3-methylimidazolium trifluoromethane-sulfonate (BMIM OTf) exhibited the best performance among the other tested ILs based on methylimidazolium.
\end{abstract}

\section{Keywords}

Organic Electrochemical Transistor (OECT), Ionic liquid (IL), PEDOT:PSS, OECT structure, printed electronics, screen printing

\footnotetext{
${ }^{1}$ Department of Technologies and Measurements / RICE, Faculty of Electrical Technologies, University of West Bohemia, 30614 Plzen, Univerzitni 26, Czech Republic

${ }^{2}$ Department of Graphic Arts and Photophysics / CEMNAT, Faculty of Chemical Technology, University of Pardubice, 53210 Pardubice, Studentska 573, Czech Republic
}

*Corresponding author, e-mail: czlukas@ket.zcu.cz

\section{Introduction}

One of the key device of organic electronics for simple electronic circuits is an organic field effect transistor (OFET) which is widely studied from the point of view of both theoretical and practical aspects [1-3]. OFETs typically operate at voltages in the order of tens of volts and lot of efforts was spent to decrease the operating voltage below $10 \mathrm{~V}$.

In contrast to OFETs, the organic electrochemical transistor (OECT) exhibits some advantageous properties, such as very low operating voltage [4], ability to work in the liquid environment [5], and simple fabrication process using common printing methods such as inkjet [6] and screen printing [7-10]. However, OECT exhibits also some disadvantages. The main one being low switching performance in terms of the switching speed and the switching ratio, which are crucial for some type of applications. The switching performance is directly proportional to the speed and efficiency of the electrochemical redox reaction in the channel between the polymer semiconductor backbone and ions injected from the gate electrode. Note that unlike OFET, the gate electrode being formed from the electrolyte is in direct contact with the channel between source and drain electrodes in the OECT. In our study we used for the channel of OECT most commonly used p-type organic polymer poly(3,4-ethylene dioxythiophene) - poly(styrene sulfonate) (PEDOT:PSS). This polymer exhibits a variety of advantageous properties such as environmental stability, good film-forming ability and the possibility to vary its electric conductivity by several orders of magnitude [11].

After the application of electric field between the gate and source (drain) electrode the ions (free to move) are injected into the channel and the electrochemical reaction starts. According to Eq. (1) cations $\mathrm{M}^{+}$are injected. Thus, $\mathrm{PEDOT}^{+}$(holes) and cations are present in the redox reaction. $\mathrm{PEDOT}^{+}$is reduced and comes into neutral "non-conducting" $\mathrm{PEDOT}^{0}$ state at the side of negative electric potential. Charge carrier concentration decreases and transistor is in the OFF state.

$$
\mathrm{PEDOT}^{+} \mathrm{PSS}^{-}+\mathrm{M}^{+}+e^{-} \leftrightarrow \mathrm{PEDOT}^{0}+\mathrm{PSS}^{-} \mathrm{M}^{+}
$$


The ratio of the drain - source currents $\left(I_{\mathrm{DS}}\right)$ in the ON and OFF states (switching ratio) and speed of switching between these states are the dominant parameters of OECT in switching applications. Their improvement is necessary to obtain OECT with satisfactory properties.

There are known two general topologies of OECT: vertical topology [12] and planar topology [13]. Vertical topology is advantageous in switching application due to the fast $I_{\mathrm{DS}}$ current response to the control gate-source voltage $\left(V_{\mathrm{GS}}\right)$ because of the shortest possible distance between the gate terminal and the channel through the thickness of the electrolyte layer. Vertical OECT with carbon drain and source contacts overlapping the channel and defining the interface between the electrolyte (mixture of PSSNa/D-sorbitol/glycerol/deionized water) and channel to limit the difference between ON and OFF times was presented [9]. The other type of vertical OECT was manufactured by using vias through the flexible substrate [10] with the poly[(quaternary imidazolium) chloride] as an electrolyte. Other interesting possibility how to fabricate the vertical OECT is using textile monofilaments coated with PEDOT:PSS, where the transistor is created on the crossing of two coated monofilaments in the presence of the electrolyte [14]. Besides the PEDOT:PSS also the poly(3-hexylthiophene) (P3HT) can be used as channel material [15]. The planar topology is mostly used in sensor applications because the electrolyte/analyte can be added to the OECT structure after the fabrication. This feature facilitates testing of different electrolytes for switching applications of OECT. The main disadvantage of this topology is slower $I_{\mathrm{DS}}$ current response to control $V_{\mathrm{GS}}$ voltage due to much larger distance between the gate terminal and the channel given by their lateral arrangement. Despite that, the planar topology is advantageous for testing of the OECT, e.g. the influence of the PEDOT:PSS channel volume [13] or the geometry [16] on the OECT performance. Planar topology is also well suitable for testing of different electrolytes [17].

The next possibility how to improve the performance of OECT, is the variation of geometrical dimensions [16]. The dimensions of channel and the distance between the channel and gate terminal through the electrolyte have the main impact on the switching parameters of OECT. Channel resistance in the ON state $\left(R_{\mathrm{ON}}\right)$ is directly proportional to the dimensions and the resistivity of the channel. Therefore, to get the high switching ratio the dimensions of the topology and materials of the channel and electrolyte must be well designed.

In this paper we present the results of the study of switching performance obtained on the planar topology of OECT [18], see Fig. 1a), and modified double GATE OECT structure, Fig. 1b). The proposed topologies are designed for the printing of all layers. The main goal of this paper is, in the first place, to find the geometry of OECT with high switching ratio, in the second place, to find suitable gate material for good transistor performance. Three different ionic liquids
(ILs), 1-ethyl-3-methylimidazolium-bis(trifluoromethyl-sulfonyl)imide (EMIM TFSI), 1-butyl-3-methylimidazolium trifluoromethane-sulfonate (BMIM OTf) and 1-ethyl-3-methylimidazolium tetrafluoroborate $\left(\mathrm{EMIM} \mathrm{BF}_{4}\right)$, were used as electrolytes in the OECT. The experimental results clearly indicate, that the OECT topology has a significant influence on its dynamic switching behavior and the chemical composition of ILs influences the electrochemical process and therefore overall switching performance of the OECT device.

\section{Materials and methods}

The OECT structures, see Fig. 1, were prepared by screen printing on a flexible substrate $\left(175 \mu \mathrm{m}\right.$ Melinex ${ }^{\circledR}$ ST504) which was used as delivered (adhesive side). At first, the channel was printed from screen printing ink formulation of PEDOT:PSS, Clevios $^{\mathrm{TM}}$ SV3, purchased from Heraeus. The PEDOT:PSS was used as delivered without further purification. The sheet resistance of the resulting layer was $\sim 700 \Omega / \square$ in the case of three electrode OECT structure, Fig. 1a) and $\sim 2.24 \mathrm{k} \Omega / \square$ in the case of double GATE OECT structure, Fig. 1b). After that, the drain, source, and gate terminals were printed from the carbon paste Gwent D1, modified by 20 wt. \% of N-methyl-2-pyrrolidone (NMP. The sheet resistance of the carbon layer was $\sim 50 \Omega / \square$. The UV curable dielectric CSP-5210 purchased from Chang Sung Corporation CSC was then printed as the mask defining an reservoir for the electrolyte. Finally, liquid electrolytes were preciously drop coated by micro pipette - $0.5 \mu$ d droplet on the three electrode OECT structure (Fig. 1a) and $0.75 \mu \mathrm{l}$ on the double GATE OECT structure (Fig. 1b). Three ionic liquids (ILs) were used as liquid electrolytes: 1-ethyl-3-methylimidazoliumbis(trifluoromethyl-sulfonyl)imide (EMIM TFSI), 1-butyl-3methylimidazolium trifluoromethane-sulfonate (BMIM OTf) and 1-ethyl-3-methylimidazolium tetrafluoroborate (EMIM $\mathrm{BF}_{4}$ ) (summarized in Table 1). They were used as delivered. The chemical structures of the ionic liquids are depicted in Fig. 2 .

Table 1 Abbreviations and full names of used ILs.

\begin{tabular}{ll}
\hline Abbreviation & Full name \\
\hline EMIM TFSI & $\begin{array}{l}\text { 1-ethyl-3-methylimidazolium- } \\
\text { bis(trifluoromethyl-sulfonyl)imide } \\
\text { 1-butyl-3-methylimidazolium } \\
\text { trifluoromethane-sulfonate }\end{array}$ \\
EMIM BF $_{4}$ & $\begin{array}{l}\text { 1-ethyl-3-methylimidazolium } \\
\text { tetrafluoroborate }\end{array}$ \\
\hline
\end{tabular}

All data were collected by the ACS BASICv2.0 software for their subsequent evaluation and visualization. 


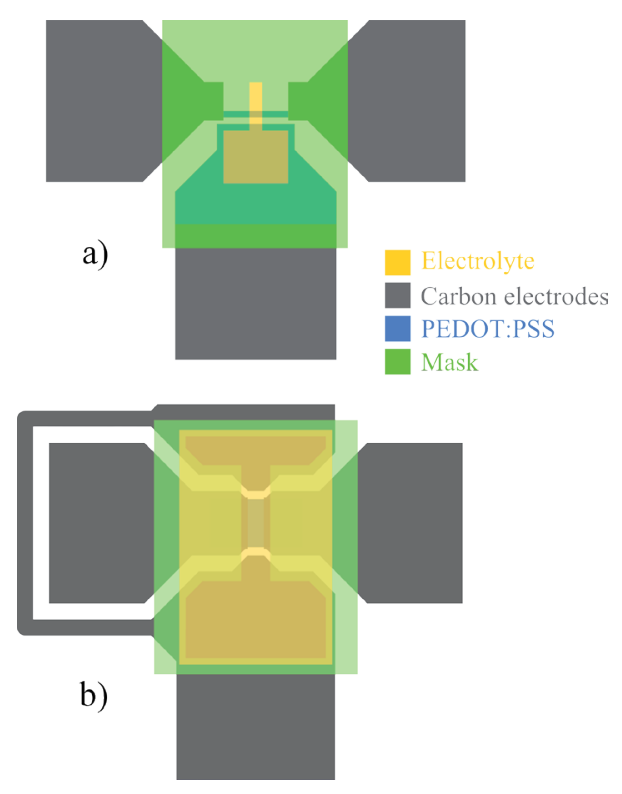

Fig. 1 The layouts of measured OECTs. a) Three electrode OECT structure, b) Double GATE OECT structure.<smiles>CCn1cc[n+](C)c1</smiles>

a)<smiles>CCCCn1cc[n+](C)c1</smiles>

b)

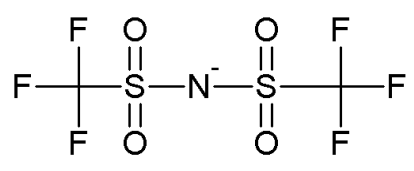

c)

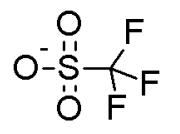

d)

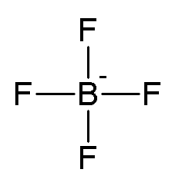

e)
Fig. 2 The chemical composition of ionic liquids used as electrolytes.

Cations (a) 1-ethyl-3-methylimidazolium $\left(\mathrm{EMIM}^{+}\right)$and (b) 1-butyl-3methylimidazolium $\left(\mathrm{BMIM}^{+}\right)$. Anions (c) bis(trifluoromethyl-sulfonyl)imide (TFSI), (d) trifluoromethanesulfonate (OTf) and (e) tetrafluoroborate $\left(\mathrm{BF}_{4}^{-}\right)$.

\section{Results}

Experimental results and extracted parameters of both OECT structures are presented in the following paragraphs. Three different ionic liquids were used to compare their influence on the switching performance of both OECT structures.

Waveforms of control $V_{\mathrm{GS}}$ voltage and response of $I_{\mathrm{DS}}$ current were measured during the testing. Switching performance parameters were estimated from the waveforms of $I_{\mathrm{DS}}$ current. The calculated characteristics were: switching ratio, $t_{\mathrm{ON} / \mathrm{OFF}}$, $t_{\mathrm{OFF} / \mathrm{ON}}, R_{\mathrm{ON}}, R_{\mathrm{OFF}}, I_{\mathrm{DSon}}$, and $I_{\mathrm{DSoff}}$. The switching ratio is the ratio between $I_{\mathrm{DS}}$ current in ON state and OFF state, Eq. (2). Response times $t_{\mathrm{ON} / \mathrm{OFF}}$ and $t_{\mathrm{OFF} / \mathrm{ON}}$ were determined as the difference between times when $I_{\mathrm{DS}}$ current reached $10 \%$ and $90 \%$ of its minimum and maximum value, Eq. (3). Resistances $R_{\mathrm{ON}}$ and $R_{\mathrm{OFF}}$ were simply calculated using the Ohm's law. $R_{\mathrm{ON}}$ was determined from the current in the $\mathrm{ON}$ state $\left(I_{\mathrm{DSon}}\right) . R_{\mathrm{OFF}}$ was dependent on the efficiency of the electrochemical reaction. Currents $I_{\mathrm{DSon}}$ and $I_{\mathrm{DSoff}}$ were determined directly from the $I_{\mathrm{DS}}$ current waveforms.

$$
\begin{gathered}
\text { Switching ratio }=\frac{I_{\mathrm{DSon}}}{I_{\mathrm{DSoff}}}(-) \\
t_{\mathrm{ON} / \mathrm{OFF}}=t_{90}-t_{10},(\mathrm{~s}) \\
t_{\mathrm{OFF} / \mathrm{ON}}=t_{10}-t_{90}
\end{gathered}
$$

\subsection{Three electrode OECT structure}

Dynamic characteristics of the three electrode OECT with different electrolytes are shown in Fig. 3 for IL EMIM TFSI, in Fig. 4 for IL BMIM OTf, and in Fig. 5 for IL EMIM BF ${ }_{4}$. All OECTs were dynamically switched by the control $V_{\mathrm{GS}}$ voltage between $-1 \mathrm{~V}$ and $1 \mathrm{~V}$ at the constant voltage $V_{\mathrm{DS}}=-1 \mathrm{~V}$. Table 2 summarizes the parameters obtained for the three electrode OECT structure.

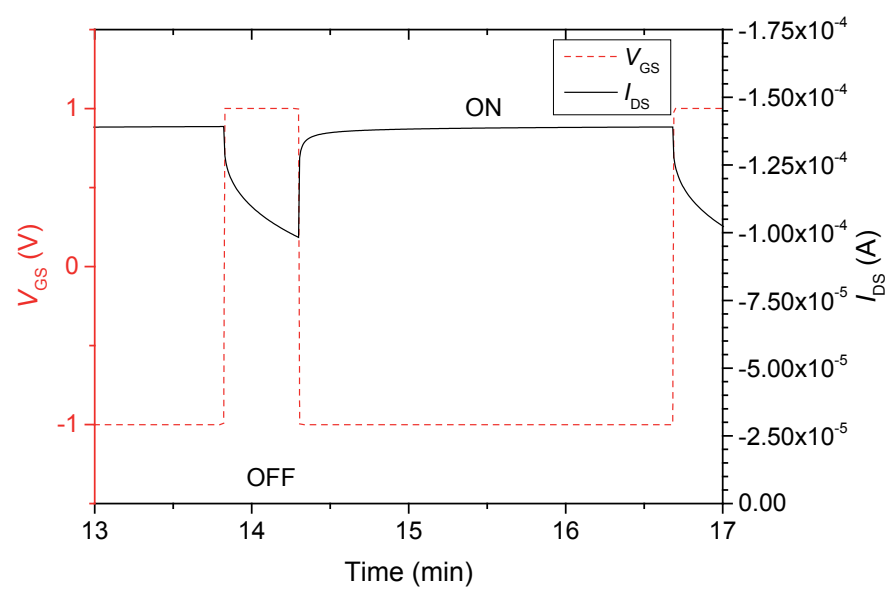

Fig. 3 Dynamic characteristics of the three electrode OECT structure with IL EMIM TFSI. The red dashed line represents electric stimulant $V_{\mathrm{GS}}$ pulse, the black solid line is the $I_{\mathrm{DS}}$ current response.

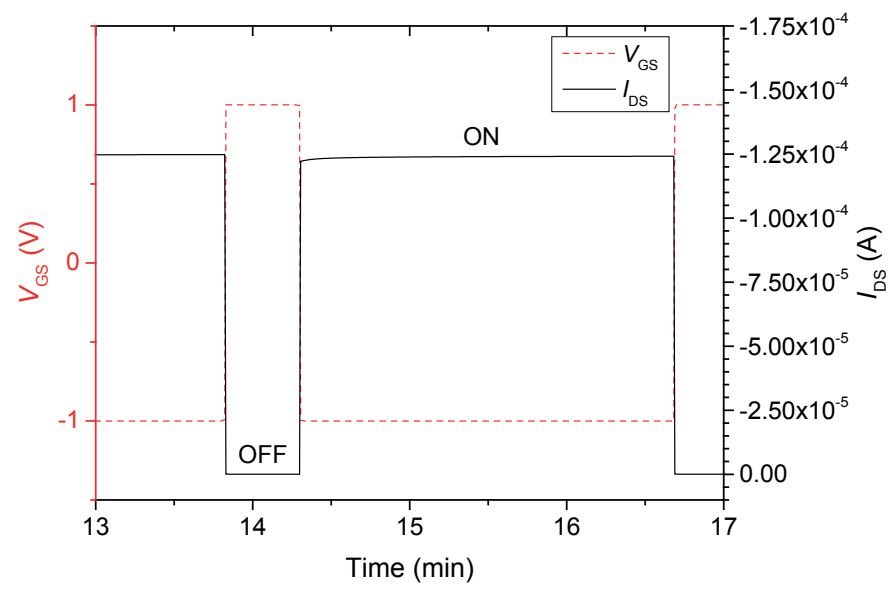

Fig. 4 Dynamic characteristics of the three electrode OECT structure with IL BMIM OTf. The red dashed line represents electric stimulant $V_{\mathrm{GS}}$ pulse, the black solid line is the $I_{\mathrm{DS}}$ current response. 


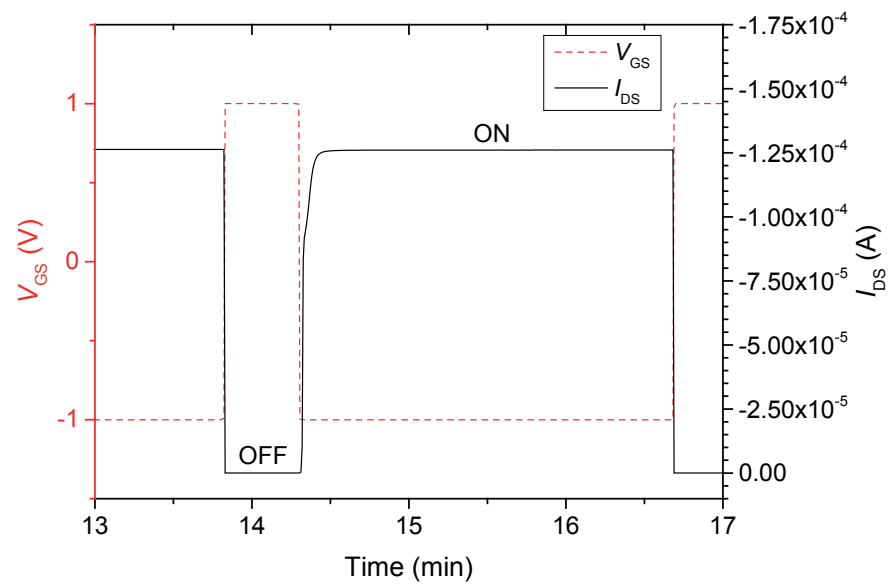

Fig. 5 Dynamic characteristics of the three electrode OECT structure with IL $\mathrm{EMIM} \mathrm{BF}_{4}$. The red dashed line represents electric stimulant $V_{\mathrm{GS}}$ pulse, the black solid line is the $I_{\mathrm{DS}}$ current response.

Table 2 Comparison of parameters extracted from the measurements of three electrode OECT structure with different electrolytes.

\begin{tabular}{llll}
\hline IL & $\begin{array}{l}\text { EMIM } \\
\text { TFSI }\end{array}$ & $\begin{array}{l}\text { BMIM } \\
\text { OTf }\end{array}$ & $\begin{array}{l}\text { EMIM } \\
\mathbf{B F}_{4}\end{array}$ \\
\hline Switching ratio $(-)$ & 1.4 & 2520 & 2440 \\
$t_{\text {ON } / \text { OFF }}(s)$ & 20.6 & 0.33 & 0.34 \\
$t_{\text {OFF } / \text { ON }}(s)$ & 8.9 & 0.34 & 3.4 \\
$R_{\text {ON }}(\Omega)$ & $7.2 \mathrm{E}+03$ & $8.0 \mathrm{E}+03$ & $7.9 \mathrm{E}+03$ \\
$R_{\text {OFF }}(\Omega)$ & $1.0 \mathrm{E}+04$ & $2.0 \mathrm{E}+07$ & $1.9 \mathrm{E}+07$ \\
$I_{\mathrm{DSon}}(\mathrm{A})$ & $-1.39 \mathrm{E}-04$ & $-1.25 \mathrm{E}-04$ & $-1.26 \mathrm{E}-04$ \\
$I_{\mathrm{DSoff}}(\mathrm{A})$ & $-9.83 \mathrm{E}-05$ & $-4.96 \mathrm{E}-08$ & $-5.16 \mathrm{E}-08$ \\
\hline
\end{tabular}

\subsection{Double GATE OECT structure}

Dynamic measurement results of the double GATE OECT structure with different electrolytes are shown in Fig. 6 for IL EMIM TFSI, in Fig. 7 for IL BMIM OTf, and in Fig. 8 for IL EMIM $\mathrm{BF}_{4}$. All OECTs were dynamically switched by the control $V_{\text {GS }}$ voltage between $0 \mathrm{~V}$ and $1 \mathrm{~V}$ at the constant voltage $V_{\mathrm{DS}}=-1 \mathrm{~V}$. Table 3 summarizes the parameters obtained for the double GATE OECT structure.

Table 3 Comparison of parameters extracted from the measurements of the double GATE OECT structure with different electrolytes.

\begin{tabular}{llll}
\hline IL & $\begin{array}{l}\text { EMIM } \\
\text { TFSI }\end{array}$ & $\begin{array}{l}\text { BMIM } \\
\text { OTf }\end{array}$ & $\begin{array}{l}\text { EMIM } \\
\mathbf{B F}_{4}\end{array}$ \\
\hline Switching ratio (-) & 8.8 & 88 & 24.0 \\
$t_{\text {ON/OFF }}(\mathrm{s})$ & 15.5 & 8.6 & 0.7 \\
$t_{\text {OFF/ON }}(\mathrm{s})$ & 38.5 & 30.9 & 68.8 \\
$R_{\mathrm{ON}}(\Omega)$ & $1.37 \mathrm{E}+3$ & $1.29 \mathrm{E}+3$ & $9.56 \mathrm{E}+3$ \\
$R_{\text {OFF }}(\Omega)$ & $1.20 \mathrm{E}+4$ & $1.24 \mathrm{E}+5$ & $5.15 \mathrm{E}+5$ \\
$I_{\mathrm{DSon}}(\mathrm{A})$ & $-7.29 \mathrm{E}-4$ & $-7.76 \mathrm{E}-4$ & $-1.05 \mathrm{E}-4$ \\
$I_{\mathrm{DS} \text { off }}(\mathrm{A})$ & $-8.31 \mathrm{E}-5$ & $-8.08 \mathrm{E}-6$ & $-1.94 \mathrm{E}-6$ \\
\hline
\end{tabular}

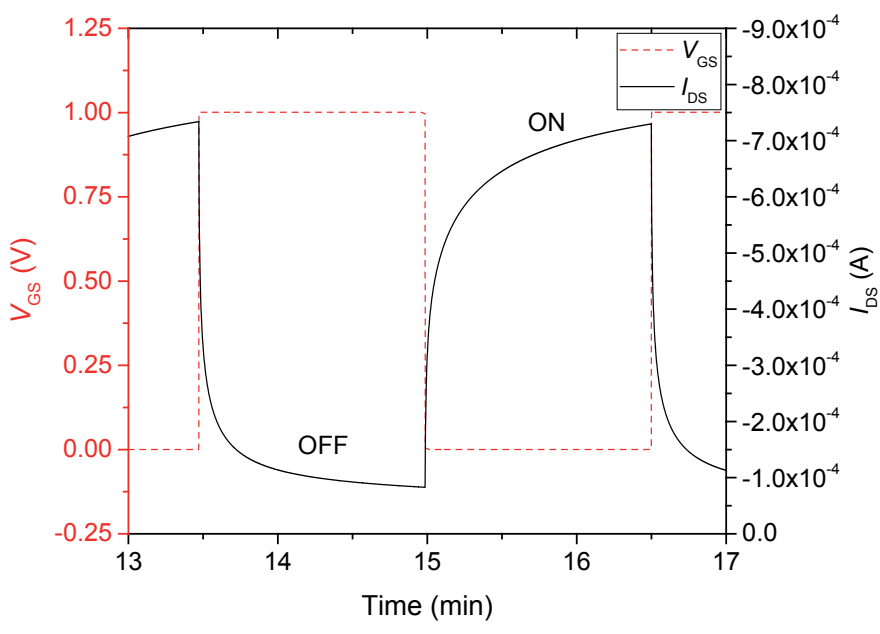

Fig. 6 Dynamic characteristics of the double GATE OECT structure with IL EMIM TFSI. The red dashed line represents electric stimulant $V_{\text {GS }}$ pulse, the black solid line is the $I_{\mathrm{DS}}$ current response.

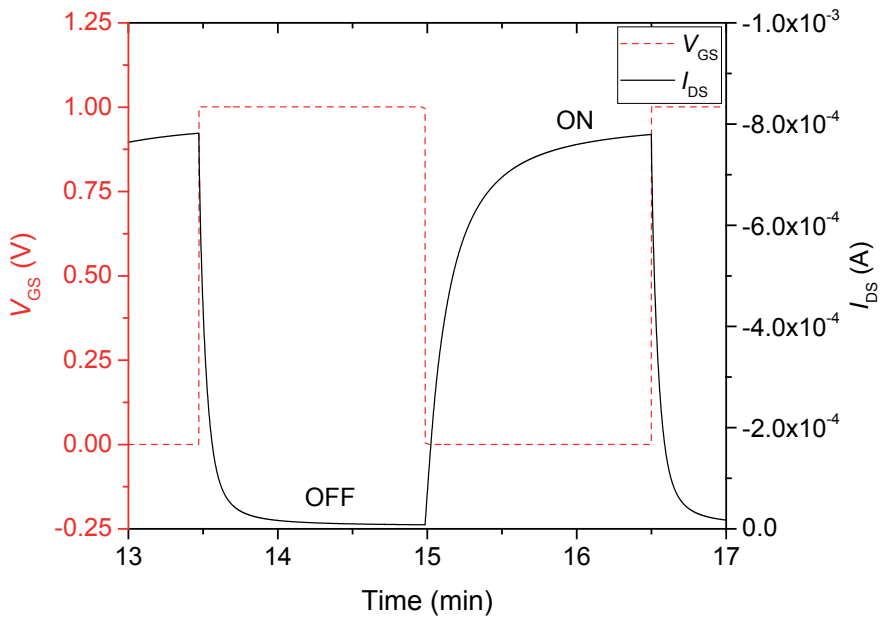

Fig. 7 Dynamic characteristics of the double GATE OECT structure with IL BMIM OTf. The red dashed line represents electric stimulant $V_{\text {GS }}$ pulse, the black solid line is the $I_{\mathrm{DS}}$ current response.

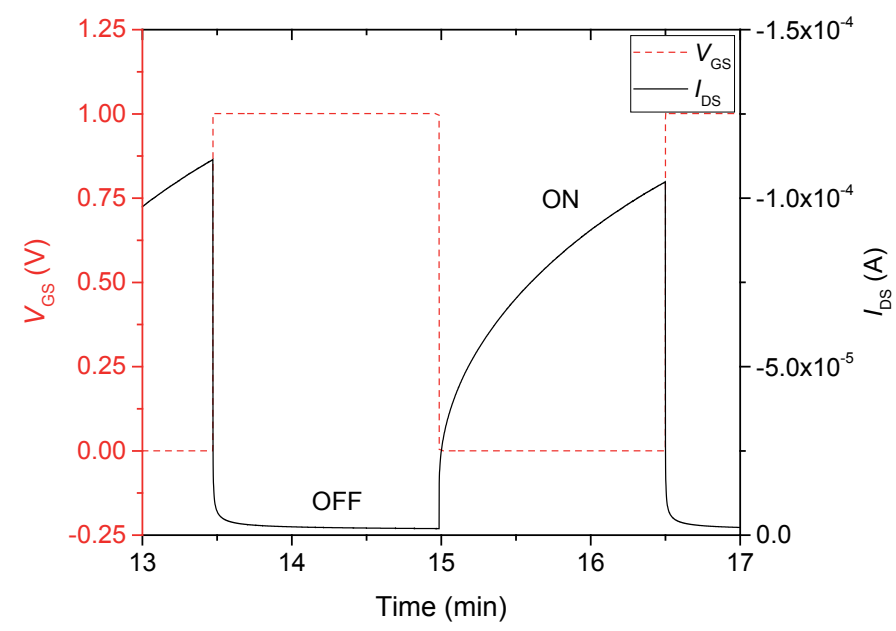

Fig. 8 Dynamic characteristics of the double GATE OECT structure with IL EMIM $\mathrm{BF}_{4}$. The red dashed line represents electric stimulant $V_{\mathrm{GS}}$ pulse, the black solid line is the $I_{\mathrm{DS}}$ current response. 


\section{Discussion}

The paper deals with the kinetics of current switching of organic electrochemical transistor (OECT) based on PEDOT:PSS hole conducting polymer. The polymer forms a transistor channel between source and drain electrodes. The gate electrode which is in the direct contact with channel polymer was prepared using several types of ionic liquids. In the initial conductive state the PEDOT is oxidized (PEDOT $\left.{ }^{+} \mathrm{PSS}^{-}\right)$ and holes represent free charge carriers. After the application of electric field between the gate and source (drain) electrodes the ions (free to move) are injected from the electrolytic gate to the channel and the electrochemical reaction starts. For negative gate bias no change of the channel current was observed. However, if the gate was positive the cations $\mathrm{M}^{+}$were injected from the ionic liquid to the channel polymer and electrochemical reaction started. The reaction is described by Eq. (1). At the negative electrode $\mathrm{PEDOT}^{+}$is reduced to neutral "nonconducting" $\mathrm{PEDOT}^{0}$, free charge carrier (positive bipolarons) concentration decreases and current is switched to low values. The transistor is in the OFF state. The ratio of the drain source currents $\left(I_{\mathrm{DS}}\right)$ in the ON and OFF states (switching ratio) and speed of switching between these states are the dominant parameters of OECT in switching applications.

Let us discuss at first the three electrode OECT structure. From Fig. 3 - Fig. 5 and Table 2 follows that the switching ratio is lowest in the case of ionic liquid EMIM TFSI (1.4). For ILs BMIM OTf and EMIM $\mathrm{BF}_{4}$ the switching ratios are nearly the same, about 2500. Similarly, the rise $\left(t_{\mathrm{ON} / \mathrm{OFF}}\right)$ and decay $\left(t_{\mathrm{OFF} / \mathrm{ON}}\right)$ times are the lowest in the case of IL EMIM TFSI (20 s and $9 \mathrm{~s}$, respectively). For BMIM OTf and EMIM $\mathrm{BF}_{4}$ both times are nearly the same, $0.3 \mathrm{~s}$. Note that decay time is in

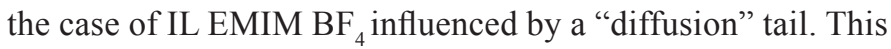
effect is very weak for IL BMIM OTf. Note that this behavior cannot be related to both electric conductivity and viscosity of ionic liquid and needs a further research.

The behavior of the transistors with the double GATE OECT structure is a little different. The main differences in the structures are shown in Table 4. It follows from Table 3 that the switching ratio is the best for transistor with IL BMIM OTf (88). The rise process is fastest for transistor with IL EMIM BF . This $_{4}$ ionic liquid shows high conductivity $\left(14 \mathrm{mS} \cdot \mathrm{cm}^{-1}\right)$ and quite low viscosity (32 $\mathrm{mPa} \cdot \mathrm{s})$, see Table 5 . However, the decay process is slower than kinetics of transistors with IL EMIM TFSI and BMIM OTf. Note that in this case the control $V_{\mathrm{GS}}$ voltage was between $0 \mathrm{~V}$ and $1 \mathrm{~V}$ (instead of $V_{\mathrm{GS}}$ between $-1 \mathrm{~V}$ and $1 \mathrm{~V}$ in the case of three electrode configuration) which could make the decay process slower.
Table 4 Comparison of channel resistance, sheet resistances, and area where the channel is in contact with IL for both measured structures.

\begin{tabular}{lll}
\hline OECT Structure & Three electrode & Double GATE \\
\hline Channel resistance $(\Omega)$ & $8.0 \mathrm{E}+3$ & $1.3 \mathrm{E}+3$ \\
Sheet resistance $(\Omega / \square)$ & $0.7 \mathrm{E}+3$ & $2.2 \mathrm{E}+3$ \\
Active area $\left(\mathrm{cm}^{2}\right)$ & $8.0 \mathrm{E}-4$ & $7.5 \mathrm{E}-3$ \\
\hline
\end{tabular}

Table 5 Parameters of used ionic liquids.

\begin{tabular}{llll}
\hline IL & $\begin{array}{l}\text { EMIM } \\
\text { TFSI }\end{array}$ & $\begin{array}{l}\text { BMIM } \\
\text { OTf }\end{array}$ & $\begin{array}{l}\text { EMIM } \\
\text { BF }_{4}\end{array}$ \\
\hline Conductivity $\left(\mathrm{mS} \cdot \mathrm{cm}^{-1}\right)$ & 8.5 & 3.4 & 14 \\
Viscosity $(\mathrm{mPa} \cdot \mathrm{s})$ & 27 & 74 & 32 \\
Density $\left(\mathrm{g} \cdot \mathrm{cm}^{-3}\right)$ & 1.5 & 1.3 & 1.3 \\
\hline
\end{tabular}

\section{Conclusion}

The selection of appropriate electrolyte together with OECT geometry are the key factors influencing the switching performance of OECT. The presented research was focused among others on the influence of ionic liquids of different chemical structures on kinetics parameters of the OECT. The following conclusions can be mentioned:

- Concerning the switching ratio the conductivity of the channel polymer (here PEDOT:PSS) must be as high as possible.

- Double GATE configuration does not improve the kinetics parameters of the transistor in comparison with the three electrode one.

- The ionic liquid with small anion exhibits shorter rise and decay times due to their higher mobility and the easer diffusion through given layers.

- After the application of the control $V_{\mathrm{GS}}$ voltage in the case of the three electrode configuration the rise part of current kinetics consists of mainly one component whereas the decay part contains in addition slow probably diffusion part.

- In the case of the double GATE configuration both the rise and decay components contain diffusion contribution - it limits the use in switching technology.

- The higher viscosity of ionic liquid the higher switching ratio of the transistor.

It could be concluded, that the three electrode OECT structure showed in general better switching performance and is therefore more suitable for systematic testing of OECT parameters than the double GATE structure. 


\section{Acknowledgement}

This research has been supported by the Ministry of Education, Youth and Sports of the Czech Republic under the RICE - New Technologies and Concepts for Smart Industrial Systems, project No. LO1607, and by the Technology Agency of the Czech Republic under the FLEXPRINT, project No. TE01020022. This work was also supported by the grant LM2015082 Centre of Materials and Nanotechnologies from the Czech Ministry of Education, Youth and Sports of the Czech Republic.

\section{References}

[1] Sirringhaus, H. "25th Anniversary Article: Organic Field-Effect Transistors: The Path Beyond Amorphous Silicon." Advanced Materials. 26(9), pp. 1319-1335. 2014. DOI: 10.1002/adma.201304346

[2] Bao, Z., Locklin, J. "Organic Field-Effect Transistors." CRC Press, Boca Raton, 2007. DOI: 10.1201/9781420008012

[3] Małachowski, M., Żmija, J. "Organic field-effect transistors." OptoElectronics Review. 18(2), pp. 121-136. 2010.

DOI: $10.2478 / \mathrm{s} 11772-010-0008-9$

[4] Khodagholy, D., Gurfinkel, M., Stavrinidou, E., Leleux, P., Herve, T., Sanaur, S., Malliaras, G. G. "High speed and high density organic electrochemical transistor arrays." Applied Physics Letters. 99(16), pp. 20112014. 2011. DOI: 10.1063/1.3652912

[5] D’Angelo, P., Coppedè, N., Tarabella, G., Romeo, A., Gentile, F., Iannotta, S., Di Fabrizio, E., Mosca, R. "Liquid electrolyte positioning along the device channel influences the operation of Organic Electro-Chemical Transistors." Organic Electronics. 15(11), pp. 3016-3023. 2014. DOI: 10.1016/j.orgel.2014.08.029

[6] Mannerbro, R., Ranlöf, M., Robinson, N., Forchheimer, R. "Inkjet printed electrochemical organic electronics." Synthetic Metals. 158(13), pp. 556-560. 2008. DOI: 10.1016/j.synthmet.2008.03.030

[7] Hütter, P. C., Rothlander, T., Scheipl, G., Stadlober, B. "All Screen-Printed Logic Gates Based on Organic Electrochemical Transistors." IEEE Transactions on Electron Devices. 62(12), pp. 4231-4236. 2015. DOI: 10.1109/TED.2015.2491342

[8] Mannerbro, R., Ranlöf, M. "Inkjet and Screen Printed Electrochemical Organic Electronics." Master Thesis, Linköping Institute of Technology, 2007. LITH-ISY-EX--07/3876--SE.

[9] Andersson Ersman, P., Nilsson, D., Kawahara, J., Gustafsson, G., Berggren, M. "Fast-switching all-printed organic electrochemical transistors." Organic Electronics. 14(5), pp. 1276-1280. 2013.

DOI: 10.1016/j.orgel.2013.02.027
[10] Kawahara, J., Andersson Ersman, P., Katoh, K., Berggren, M. "Fastswitching printed organic electrochemical transistors including electronic vias through plastic and paper substrates." IIEEE Transactions on Electron Devices. 60(6), pp. 2052-2056. 2013.

DOI: $10.1109 /$ TED.2013.2258923

[11] Knoll, M., Thämer, M. "An enhancement-mode electrochemical organic field-effect transistor." Electrochemistry Communications. 13(6), pp. 597-599. 2011. DOI: 10.1016/j.elecom.2011.03.019

[12] Khodagholy, D., Rivnay, J., Sessolo, M., Gurfinkel, M., Leleux, P., Jimison, L. H., Stavrinidou, E., Herve, T., Sanaur, S., Owens R. M., Malliaras, G. G. "High transconductance organic electrochemical transistors." Nature Communications. 4, 2133. 2013. DOI: 10.1038/ncomms3133

[13] Hütter, P. C., Fian, A., Gatterer, K., Stadlober, B. "Efficiency of the Switching Process in Organic Electrochemical Transistors." AACS Applied Materials \& Interfaces. 8(22), pp. 14071-14076. 2016. DOI: $10.1021 /$ acsami.6b02698

[14] Hamedi, M., Forchheimer, R., Inganäs, O. "Towards woven logic from organic electronic fibres." Nature Materials. 6(5), pp. 357-362. 2007. DOI: $10.1038 /$ nmat1884

[15] Braga, D., Erickson, N. C., Renn, M. J., Holmes, R. J., Frisbie, C. D. "High-transconductance organic thin-film electrochemical transistors for driving low-voltage red-green-blue active matrix organic light-emitting devices." Advanced Functional Materials. 22(8), pp. 1623-1631. 2012. DOI: 10.1002/adfm.201102075

[16] Hütter, P. C., Rothländer, T., Haase, A., Trimmel, G., Stadlober, B. "Influence of geometry variations on the response of organic electrochemical transistors." Applied Physics Letters. 103(4), pp. 15-19. 2013. DOI: $10.1063 / 1.4816781$

[17] Yi, Z., Natale, G., Kumar, P., Di Mauro, E., Heuzey, M.-C., Soavi, F., Perepichka, I. I., Varshney, S. K., Santato, C., Cicoira, F. "Ionic liquidwater mixtures and ion gels as electrolytes for organic electrochemical transistors." Journal of Materials Chemistry C. 3(25), pp. 6549-6553. 2015. DOI: 10.1039/C5TC00712G

[18] Mracek, L., Pretl, S., Syrovy, T., Hamacek, A. "Ionic liquid as an electrolyte for organic electrochemical transistor." In: 2015 38th International Spring Seminar on Electronics Technology (ISSE), Eger, May 6-10, 2015, pp. 11-15. DOI: 10.1109/ISSE.2015.7247952 